\title{
Complexities in the estimation of overdiagnosis in breast cancer screening
}

\author{
SW Duffy ${ }^{*,}$, E Lynge², H Jonsson ${ }^{3}$, S Ayyaz' and AH Olsen' \\ 'Cancer Research UK Centre for Epidemiology Mathematics and Statistics, Wolfson Institute of Preventive Medicine, Charterhouse Square, London ECI M \\ 6BQ UK; ${ }^{2}$ Institute of Public Health, University of Copenhagen, Øster Farimagsgade 5, opg. B, Copenhagen K DK-I OI4, Denmark; ${ }^{3}$ Department of \\ Radiation Sciences, Oncology, Umeå University, Umeå, Sweden
}

There is interest in estimating and attributing temporal changes in incidence of breast cancer in relation to the initiation of screening programmes, in particular to estimation of overdiagnosis of breast cancer as a result of screening. In this paper, we show how screening introduces complexities of analysis and interpretation of incidence data. For example, lead time brings forward time- and age-related increases in incidence. In addition, risk factors such as hormone replacement therapy use have been changing contemporaneously with the introduction of screening. Although we do not indicate exactly how such complexities should be corrected for, we use some simple informal adjustments to show how they may account for a substantial proportion of increased incidence, which might otherwise erroneously have been attributed to overdiagnosis. We illustrate this using an example of analysis of breast cancer incidence data from Sweden.

British Journal of Cancer (2008) 99, I I76- | I78. doi:I0.1038/sj.bjc.6604638 www.bjcancer.com

Published online 2 September 2008

(c) 2008 Cancer Research UK

Keywords: mammography; breast cancer screening; overdiagnosis

In the 1990s, many countries experienced substantial increases in breast cancer incidence (Chu et al, 1996; Botha et al, 2003), whereas since 2002, it has fallen in the USA (Ravdin et al, 2007), both being subjects of the study (Glass et al, 2007). The recent decline was mostly attributed to a lower prevalence of hormone replacement therapy (HRT) use (Ravdin et al, 2007), whereas the levelling off of screening mammography rates in the late 1990s has been suggested as contributing (Glass et al, 2007). In relation to increased incidence in the 1990s, this is usually attributed to the introduction of mammographic screening (Jonsson et al, 2005; Seppänen et al, 2006). It is well established that this causes an immediate rise in incidence, mainly due to the early diagnosis of a many prevalent asymptomatic cancers (Jonsson et al, 2005; Svendsen et al, 2006). Also important is overdiagnosis, that is, the detection by screening of cancer which would never have been diagnosed in the host's lifetime if screening had not taken place (Olsen et al, 2006; Paci et al, 2006; Biesheuvel et al, 2007), estimated rates of overdiagnosis, ranging from around 3 to $50 \%$ or more (Biesheuvel et al, 2007).

In estimating overdiagnosis either in primary research or in reviews, the complexities in interpreting cancer incidence data in the epoch of screening are important (Paci and Duffy, 2005). For example, one of the present authors (Jonsson et al, 2005) identified an excess incidence in the screening epoch in Sweden, which remains unexplained, but specifically did not attribute this to overdiagnosis. Indeed, the authors cited a number of other influences, including the prevalence screen effect mentioned above

*Correspondence: Dr. SW Duffy; E-mail: stephen.duffy@cancer.org.uk Received 4 April 2008; revised 23 June 2008; accepted I August 2008; published online 2 September 2008 and potential changes in risk-factor prevalence, notably the use of HRT. Nevertheless, certain studies have interpreted the above results as direct estimates of overdiagnosis (Biesheuvel et al, 2007; Glass et al, 2007).

This potential overinterpretation of incidence patterns is symptomatic of the difficulty in estimating overdiagnosis in the context of disease screening. Before screening was initiated, breast cancer incidence was increasing in many countries (Ravdin et al, 2007). However, the complex interaction among increasing incidence, lead time, age at diagnosis and calendar period of diagnosis is often poorly appreciated (Paci and Duffy, 2005). Here, we describe the complexities of the incidence/screening relationship and illustrate their potential to explain substantial increases in incidence, using one study as an illustration (Jonsson et al, 2005).

\section{MATERIALS AND METHODS}

\section{Screening and incidence}

In the first instance, it should be noted that before the introduction of screening, breast cancer incidence was rising in many parts of the world (Pisani, 1992) so that any estimate of excess incidence associated with screening should take such trends into account (Jonsson et al, 2005). In addition, changes in riskfactor prevalences contemporaneous with the advent of screening may add to underlying incidence beyond the prescreening time trend. A prime example of this is the two- to threefold increase in HRT use in Sweden between the early 1980s and the early 1990s (Søgaard et al, 2000). As we shall see below, the interrelationship between time trends and the effect of screening can be complicated. 
When a screening programme is introduced, and all members of a target population are offered screening for the first time, a major rise in incidence is observed. The randomised trials show that around 3 years of incidence is harvested at the first screen in women aged 50 or above (Tabar et al, 1992). Thus, if it takes 2 years to carry out the prevalence screen, around a $100 \%$ increase in incidence will be observed during the period. This is an important component of the evidence for the estimated duration of the preclinical screen-detectable period, which is typically 2-4 years (Paci and Duffy, 1991; Tabar et al, 1992). This is largely not overdiagnosis, but early diagnosis, as is evidenced by the fact that incidence of interval cancers in the screened population thereafter is lower than the expected total incidence in the absence of screening (Tabar et al, 1992). The interpretation (Biesheuvel et al, 2007) of one reported prevalence screen effect (Jonsson et al, 2005) as 'overdetection' (Biesheuvel et al, 2007) indicates that this is still not fully appreciated.

What is even less well appreciated is the fact that a lead-time effect persists beyond the introductory phase of screening. Even if there were no overdiagnosis at all, the incidence would not return to, or fall below, that expected in the absence of screening until after the cohort screened has passed beyond the upper age limit for screening. This is due to several factors, but three of them are particularly often neglected:

(1) Prevalence screening of women reaching the lower age limit of the screening programme. Seven years or more after the start of screening in Sweden, a $54 \%$ increase of breast cancer incidence was observed at ages 50-59 (Jonsson et al, 2005). Several Swedish counties, however, started screening at age 50, so this will not be purely incidence screening, and there will also be prevalence screening of women aged 50-51, a point raised in this paper.

(2) Increased age-specific incidence due to lead time. If screening confers an average lead time of 3 years on screen-detected cancers and two-thirds of cancers diagnosed in the programme are screen detected, the average lead time in the programme will be 2 years. This means that age 52 incidence will be observed at age 50, age 53 incidence at age 51 and so on. As incidence increases with age, this will artificially increase the observed age-specific incidence.

(3) Increased incidence due to anticipation of the temporal trend. As noted above, in almost all countries where mammographic screening is common, breast cancer incidence was increasing over time before the programme started. With a 2-year average lead time, we will observe 1995 incidence in 1993, 1996 incidence in 1994 and so on, again artificially inflating the observed incidence.

Figure 1 shows the incidence of breast cancer in England and Wales in 1985 and 1995, plus a third line representing the expected incidence in 1995 based on incidence trends in 1980-86, before screening was started. The first intuitive interpretation of such a graph is to interpret the vertical differences as increased incidence. However, in the age range covered by screening, 50-64, it is instructive to consider the horizontal differences. These will be a product of the three lead-time phenomena noted above, together with genuine underlying changes in risk-factor prevalence which may have caused an increased incidence with time, and overdiagnosis. The important point to note is that estimation of overdiagnosis should take into account the lead-time phenomena in addition to overdiagnosis and changes in incidence.

\section{Example - breast cancer incidence in Sweden, ages 50-69}

In the study of incidence in eleven counties in Sweden, with a target population for screening of 463405 women, it was estimated that 7 years or more after the initiation of screening at ages $50-59$,

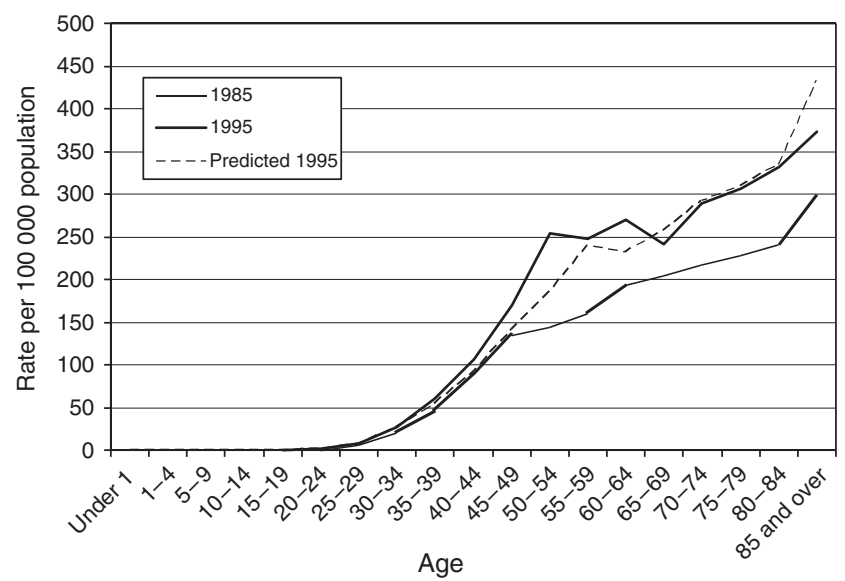

Figure I Age-specific breast cancer incidence in the UK in 1985, before the screening programme, and 1995, during the screening programme, with expected incidence in 1995 calculated from incidence trends observed before the breast screening programme began.

the breast cancer incidence relative to that expected from prescreening trends was 1.69 , a $69 \%$ excess (Jonsson et al, 2005). After a lead-time adjustment involving moving some cases in the age group 40-49 to the 50-59 group and some from the 50-59 to the 60-69 age group, the relative incidence was 1.54 .

\section{RESULTS}

To consider the possible contribution to the raw figure of 1.69 of some of the complexities referred to above, we first remove the effects of the prevalence screening of those reaching age 50 . In the prevalent phase, the excess incidence was $84 \%$. The counties starting screening at age 50 comprised around $26 \%$ of the population, and within these counties, $20 \%$ of the screens at ages 50-59 were estimated to be prevalence screens. If $x$ denotes the increased incidence excluding prevalence screens, we have:

$$
0.26 \times 0.2 \times 1.84+(1-0.26 \times 0.2) \times x=1.69
$$

This solves to give $x=1.68$, a very minor change.

Next, with respect to the effect of lead time on age-specific incidence, incidence in the absence of screening increases by around $64 \%$ between age groups 50-59 and 60-69 (Jonsson et al, 2005). A 2.4-year lead time as estimated in that study for this age group therefore corresponds to a $13 \%$ increase in incidence. Dividing 1.68 by 1.13 gives a figure of 1.49 . This method implicitly replaces the incidence in the age group $50-59$ by that in the group 2.4 years older than this, without physically shifting cases between age groups in the analysis.

To consider an adjustment for use of HRT, this increased the female population as a whole from around $1.2 \%$ to around $4.0 \%$ between 1985 and 1995 (Søgaard et al, 2000). If we assume that around half of HRT use occurred in the $13 \%$ of the population aged $50-59$, this would imply an increase from 4.6 to $15.3 \%$ in the period. Using the estimated relative risk of 1.66 with current HRT use from the million women study (Beral et al, 2003), and a method for aggregate exposure measures (Duffy et al, 2007), this would mean a $7 \%$ increase in risk due to HRT; dividing the 1.49 by 1.07 gives a relative incidence of 1.39 , a $39 \%$ excess.

\section{DISCUSSION}

We have described some of the complexities of interpretation and analysis in estimation of overdiagnosis in breast cancer screening, 
and illustrated these with an example from Sweden (Jonsson et al, 2005). The $39 \%$ excess is considerably smaller than the $54 \%$ estimated by the lead-time adjustment of simply shifting of a number of cases by age group. It is still a rough approximation, and we do not claim that it is the correct estimate of overdiagnosis. It relies on a number of assumptions, and is based on invasive cancer incidence alone: inclusion of ductal carcinoma in situ might give larger estimates of excess incidence. It does, however, illustrate that adjustment for some of the complexities in incidence by time and age and in screening-induced lead time can account for a substantial proportion of any observed excess incidence coincident with screening, before any consideration of overdiagnosis is necessary. It also suggests that the results of the Swedish study have been overinterpreted as overdiagnosis estimates by others (Biesheuvel et al, 2007; Glass et al, 2007). Thus, there are also reasons for believing that much of the $69 \%$ uncorrected excess observed at ages 50-59 in the 7 years postscreening epoch is largely due to other factors than overdiagnosis. Also, after the prevalence period, the incidence fell dramatically, as might be expected, but then equally dramatically rose again to the prevalence level over the following 6 years (Jonsson et al, 2005). It is difficult to see how overdiagnosis rates should fall and then rise again in this manner.

The fact that lead time and related phenomena are likely to continue to inflate the observed incidence until after the cohort has ceased to be screened for some time implies that cumulative incidence in screened or invited cohorts compared to unscreened or uninvited is a desirable source of data for estimation of overdiagnosis. Using cumulative incidence in the Malmö Trial study and control groups, followed up until after screening had ceased in the study group (Zackrisson et al, 2006), overdiagnosis rates of $7-8 \%$ were estimated, considerably more plausible than observational incidence estimates which did not take full account of the extent of current and previous screening exposure in the cohorts studied (Zahl et al, 2004). There are problems of interpretation of Zackrisson's results, notably the relatively recent cessation of screening in one age group, but the results are certainly more consistent with the disease progression modelling which explicitly takes lead time into account (Olsen et al, 2006).

Finally, we note that overdiagnosis in this context is an epidemiological rather than a pathological concept, the definition of disease concerning what would not have been diagnosed if the screening had not taken place. It therefore is not necessarily restricted to in situ or 'minimal' invasive cancer, although intuitively one would expect that the majority of overdiagnosed cases would be in one of these two categories. Definitive estimation of overdiagnosis of invasive and in situ carcinoma in breast screening remains to be done. This paper emphasises that any such definitive estimation needs to take into account the complexities of the relationship between screening lead time and patterns of incidence by time and age. It is likely that after adjusting for these, overdiagnosis estimates will be smaller than many rates quoted in the past. The challenge for the future will be the avoidance of overtreatment of screen-detected and early stage cancers.

\section{REFERENCES}

Beral V, Million Women Study Collaborators (2003) Breast cancer and hormonereplacement therapy in the Million Women Study. Lancet 362: 419-427

Biesheuvel C, Barratt A, Howard K, Houssami N, Irwig L (2007) Effects of study methods and biases on estimates of invasive breast cancer overdetection with mammography screening: a systematic review. Lancet Oncol 8: $1129-1138$

Botha JL, Bray F, Sankila R, Parkin DM (2003) Breast cancer incidence and mortality trends in 16 European countries. Eur J Cancer 39: 1718-1729

Chu KC, Tarone RE, Kessler LG, Ries LA, Hankey BF, Miller BA, Edwards BK (1996) Recent trends in US breast cancer incidence, survival, and mortality rates. J Natl Cancer Inst 88: 1571 - 1579

Duffy SW, Jonsson H, Agbaje OF, Pashayan N, Gabe R (2007) Avoiding bias from aggregate measures of exposure. J Epidemiol Community Health 61: $461-463$

Glass AG, Lacey Jr JV, Carreon JD, Hoover RN (2007) Breast cancer incidence, 1980-2006: combined roles of menopausal hormone therapy, screening mammography, and estrogen receptor status. J Natl Cancer Inst 99: $1152-1161$

Jonsson H, Johansson R, Lenner P (2005) Increased incidence of invasive breast cancer after the introduction of service screening with mammography in Sweden. Int J Cancer 117: 842-847

Olsen AH, Agbaje OF, Myles JP, Lynge E, Duffy SW (2006) Overdiagnosis, sojourn time and sensitivity in the Copenhagen mammography screening programme. Breast J 12: $338-342$

Paci E, Duffy SW (1991) Modelling the analysis of breast cancer screening programmes: sensitivity, lead time and predictive value in the Florence District Programme (1975-1986). Int J Epidemiol 20: $852-858$

Paci E, Duffy SW (2005) Overdiagnosis and overtreatment of breast cancer: overdiagnosis and overtreatment in service screening. Breast Cancer Res 7: $266-270$
Paci E, Miccinesi G, Puliti D, Baldazzi P, De Lisi V, Falcini F, Cirilli C, Ferretti S, Mangone L, Finarelli AC, Rosso S, Segnan N, Stracci F, Traina A, Tumino R, Zorzi M (2006) Estimate of overdiagnosis of breast cancer due to mammography after adjustment for lead time. A service screening study in Italy. Breast Cancer Res 8: R68

Pisani P (1992) Breast cancer: geographic variation and risk factors. J Environ Pathol Toxicol Oncol 11: 313-316

Ravdin PM, Cronin KA, Howlader N, Berg CD, Chlebowski RT, Feuer EJ, Edwards BK, Berry DA (2007) The decrease in breastcancer incidence in 2003 in the United States. $N$ Engl J Med 356: $1670-1674$

Seppänen J, Heinävaara S, Antilla A, Sarkeala T, Virkkunen H, Hakulinen T (2006) Effects of different phases of an invitational screening program on breast cancer incidence. Int J Cancer 119: 920-924

Søgaard AJ, Tollan A, Berntsen GK, Fønnebø V, Magnus JH (2000) Hormone replacement therapy: knowledge, attitudes, self-reported use - and sales figure in Nordic women. Maturitas 35: 201-214

Svendsen AL, Olsen AH, von Euler-Chelpin M, Lynge E (2006) Breast cancer incidence after the introduction of mammography screening: what should be expected? Cancer 106: $1883-1890$

Tabar L, Fagerberg G, Duffy SW, Day NE, Gad A, Grontoft O (1992) Update of the Swedish two-county program of mammographic screening for breast cancer. Radiol Clin North Am 30: 187-210

Zackrisson S, Andersson I, Janzon L, Manjer J, Garne JP (2006) Rate of over-diagnosis of breast cancer 15 years after end of Malmö mammographic screening trial: follow-up study. BMJ 332: 689-692

Zahl PH, Strand BH, Mæhlen J (2004) Incidence of breast cancer in Norway and Sweden during introduction of nationwide screening: prospective cohort study. BMJ 328: 921 - 924

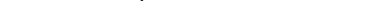

